Relations industrielles

Industrial Relations

\title{
Managers and Management in West Germany, by Peter \\ Lawrence, London, Croom Helm, 1980, 202 pp., ISBN \\ 0-85664-912-0.
}

\section{Michel Paquin}

Volume 38, numéro 2, 1983

URI : https://id.erudit.org/iderudit/029372ar

DOI : https://doi.org/10.7202/029372ar

Aller au sommaire du numéro

Éditeur(s)

Département des relations industrielles de l'Université Laval

ISSN

0034-379X (imprimé)

1703-8138 (numérique)

Découvrir la revue

Citer ce compte rendu

Paquin, M. (1983). Compte rendu de [Managers and Management in West Germany, by Peter Lawrence, London, Croom Helm, 1980, 202 pp., ISBN 0-85664-912-0.] Relations industrielles / Industrial Relations, 38(2), 455-456. https://doi.org/10.7202/029372ar

Tous droits réservés @ Département des relations industrielles de l'Université Laval, 1983
Ce document est protégé par la loi sur le droit d'auteur. L'utilisation des services d’Érudit (y compris la reproduction) est assujettie à sa politique d'utilisation que vous pouvez consulter en ligne.

https://apropos.erudit.org/fr/usagers/politique-dutilisation/ 
ont un intérêt commun et égal à entreprendre ces expériences, Kelly démontre que les coûts de la restructuration des tâches sont plus élevés pour les employés: emplois perdus, «intensification» du travail, responsabilité accrue, imputabilité, tandis que les avantages qu'ils en retirent censément sont d'ordre psychologique (les avantages des employeurs sont d'ordre économique).

On comprend mieux dans ces conditions les réticences des syndicats à l'égard des diverses formes de restructuration des tâches. L'analyse minutieuse de Kelly met en lumière les principes de rationalisation du travail qui guident ces expériences au-delà du vocabulaire progressiste qui les recouvre. D'ailleurs, l'auteur suggère de dépouiller ce vocabulaire et de remplacer à l'avenir l'expression «groupes autonomes» par "groupes flexibles» et l'expression "enrichissement des tâches» par «intégration verticale des rôles». Quant à l'avenir proprement dit de la restructuration des tâches, Kelly refuse d'adhérer à l'idée voulant qu'il s'agisse d'un phénomène transitoire, bientôt à son déclin. Les employeurs auront toujours intérêt à rationaliser le travail et à diminuer les coûts pour répondre aux impératifs du marché et la concurrence. Des formes de réorganisation du travail qui contribueront à augmenter la productivité subsisteront et se développeront sans cesse.

Alain VINET

Université Laval

Managers and Management in West Germany, by Peter Lawrence, London, Croom Helm, 1980, 202 pp., ISBN 0-85664-912-0

$\mathrm{Au}$ moment où bon nombre d'entreprises américaines connaissent des difficultés importantes, il est de bon ton d'examiner les pratiques de gestion qui ont cours dans des pays où, semble-t-il, les entreprises mondiamieux la crise qui frappe l'économie mondiale. Parmi les pays à la mode, on note le Japon, mais aussi l'Allemagne.
Peter Lawrence est un sociologue anglais qui, dans son ouvrage Managers and management in West Germany nous livre une étude très poussée des managers et de la gestion des entreprises en Allemagne fédérale. Tout en décrivant fort bien l'expérience allemande, l'auteur fait aussi, à l'occasion, des comparaisons avec la situation prévalant dans d'autres pays, notamment en Angleterre et aux États-Unis. Cette approche comparative rend encore plus intéressante la lecture de ce livre qui, par ailleurs, est fort bien construit.

Dans un premier chapitre, l'auteur présente les principales données sur la place de l'Allemagne fédérale parmi les grandes puissances industrielles et dresse un tableau rapide de l'évolution économique de ce pays depuis la fin de la seconde guerre et de sa situation politique et sociale.

Dans les chapitres suivants (deux à huit) l'auteur traite successivement de la structure des entreprises allemandes (forme légale, taille, participation des travailleurs), du «background» des managers allemands (milieu social, qualifications), du caractère du management allemand (aspect collégial, techniques utilisées, etc.), des vues des managers allemands sur l'entreprise et leur "profession" d'administrateur, de la gestion de la production, du contremaître et, enfin, du statut de l'industrie dans la société allemande.

Parmi les observations les plus intéressantes de l'auteur, mentionnons que les managers allemands ne sont pas des diplômés d'écoles de gestion puisqu'il n'y a pas d'institutions de ce type en allemagne. Une grande proportion des managers allemands sont diplômés universitaires et la majorité d'entre eux sont des ingénieurs. La place des ingénieurs dans l'entreprise allemande reflète l'importance qu'on accorde à la production qui a un statut très élevé. Conception du produit, qualité du produit, respect des délais de livraison et service après vente sont les points forts de l'entreprise allemande. L'importance accordée à la «technik» fait que le manager comprend la production. De leur côté, les tra- 
vailleurs allemands reçoivent un apprentissage de trois ans, période pendant laquelle ils vont surtout travailler dans une entreprise, afin de pouvoir accéder à un métier donné. Les contremaîtres, en plus d'une formation d'apprenti, doivent recevoir une formation de «Meister».

Les qualifications élevées des ouvriers, des contremaîtres et des gérants de la production facilitent entre ces groupes des relations reposant sur la confiance. Dans un tel système, les employés sont respectés et considérés comme importants. Aussi, il n'est pas surprenant de constater que l'aliénation au travail ne soit pas une préoccupation du travailleur allemand qui bénéficie d'un travail plus «enrichi» que les travailleurs d'autres pays.

L'auteur termine son ouvrage par un chapitre très intéressant consacré à la possibi- lité d'exporter le modèle allemand. La gestion des entreprises est liée à tout un contexte économique, politique, social, culturel, juridique et technique. Il est difficile, lorsqu'on examine une expérience étrangère, d'analyser logiquement ce qui arriverait si on en implantait certains éléments dans une autre culture. Ceci ne veut pas dire qu'on ne peut tirer de leçons utiles de l'étude d'autres pays. On peut s'inspirer d'expériences étrangères, mais on ne dispose d'aucun moyen pour connaître à l'avance les résultats.

Le livre de Peter Lawrence est extrêmement intéressant. Il mérite d'être lu par toute personne qui s'intéresse à l'administration comparée.

Michel PAQUIN

École nationale

d'administration publique, Montréal 